\title{
Well-posedness of delay parabolic difference equations
}

\author{
Allaberen Ashyralyev ${ }^{1,2^{*}}$ and Deniz Agirseven ${ }^{3}$
}

"Correspondence: aashyr@fatih.edu.tr

${ }^{1}$ Department of Mathematics, Fatih University, Istanbul, 34500, Turkey

2Department of Mathematics, ITTU, Gerogly Street, Ashgabat, 74400,

Turkmenistan

Full list of author information is available at the end of the article

\begin{abstract}
The well-posedness of difference schemes of the initial value problem for delay differential equations with unbounded operators acting on delay terms in an arbitrary Banach space is studied. Theorems on the well-posedness of these difference schemes in fractional spaces are proved. In practice, the coercive stability estimates in Hölder norms for the solutions of difference schemes of the mixed problems for delay parabolic equations are obtained.
\end{abstract}

Keywords: well-posedness; delay parabolic equations; fractional spaces; coercive stability

\section{Introduction}

Approximate solutions of the delay differential equations have been studied extensively in a series of works (see, for example, [1-6] and the references therein) and developed over the last three decades. In the literature mostly the sufficient condition

$$
|b(t)| \leq \operatorname{Re} a(t), \quad t \geq 0
$$

was considered for the stability of the following test delay differential equation:

$$
\frac{d v(t)}{d t}+a(t) v(t)=b(t) v(t-\omega), \quad t>0
$$

with the initial condition

$$
v(t)=g(t) \quad(-\omega \leq t \leq 0) .
$$

It is known that delay differential equations can be solved by applying standard numerical methods for ordinary differential equations without the presence of delay. However, it is difficult to generalize any numerical method to obtain a high order of accuracy algorithms, because high-order methods may not lead to efficient results. It is well known that even if $a(t), b(t)$ and $g(t)$ are arbitrary differentiable functions, $v(t)$ may not possess the higher-order derivatives for a sufficiently large $t$. Therefore, we have non-smooth solution of delay differential equations for given smooth data. This is the main difficulty in the study of the convergence of numerical methods for delay differential equations.

() 2014 Ashyralyev and Agirseven; licensee Springer. This is an Open Access article distributed under the terms of the Creative Commons Attribution License (http://creativecommons.org/licenses/by/2.0), which permits unrestricted use, distribution, and reproduction in any medium, provided the original work is properly cited. 
Delay partial differential equations arise from various applications, like in climate models, biology, medicine, control theory, and many others (see, for example, [7] and the references therein).

The theory of approximate solutions of delay partial differential equations has received less attention than delay ordinary differential equations. A situation which occurs in delay partial differential equations when the delay term is an operator of lower order with respect to the other operator term is widely investigated (see, for example, [7-9] and the references therein). In the case where the delay term is an operator of the same order with respect to other operator term, this is studied mainly in a Hilbert space (see, for example, [10] and the references therein). In fact there are very few papers where the delay term is an operator of the same order with respect to the other operator term, this being investigated in a general Banach space (see [11-14]) and in these works, the authors look only for partial differential equations under regular data. Additionally, approximate solutions of the delay parabolic equations in the case where the delay term is a simple operator of the same order with respect to the other operator term were studied recently in papers [15-19].

It is known that various initial-boundary value problems for linear evolutionary delay partial differential equations can be reduced to an initial value problem of the form

$$
\left\{\begin{array}{l}
\frac{d v(t)}{d t}+A v(t)=B(t) v(t-\omega)+f(t), \quad t \geq 0 \\
v(t)=g(t) \quad(-\omega \leq t \leq 0)
\end{array}\right.
$$

in an arbitrary Banach space $E$ with the unbounded linear operators $A$ and $B(t)$ in $E$ with dense domains $D(A) \subseteq D(B(t))$. Let $A$ be a strongly positive operator, i.e. $-A$ is the generator of the analytic semigroup $e^{-t A}(t \geq 0)$ of the linear bounded operators with exponentially decreasing norm when $t \rightarrow \infty$. That means the following estimates hold:

$$
\left\|e^{-t A}\right\|_{E \mapsto E} \leq M e^{-\delta t}, \quad\left\|t A e^{-t A}\right\|_{E \mapsto E} \leq M, \quad t>0
$$

for some $M>1, \delta>0$. Let $B(t)$ be closed operators.

The strongly positive operator $A$ defines the fractional spaces $E_{\alpha}=E_{\alpha}(A, E)(0<\alpha<1)$ consisting of all $u \in E$ for which the following norms are finite:

$$
\|u\|_{E_{\alpha}}=\sup _{\lambda>0}\left\|\lambda^{1-\alpha} A e^{-\lambda A} u\right\|_{E}
$$

As noted in [19], it is important to study the stability of solutions of the initial value problem (4) for delay differential equations and of difference schemes for approximate solutions of problem (4) under the assumption that

$$
\left\|B(t) A^{-1}\right\|_{E \mapsto E} \leq 1
$$

holds for every $t \geq 0$. This assumption for the delay differential equation (2) follows from assumption (1) in the case when $E=\mathbb{R}^{1}$. Unfortunately, we have not been able to obtain the stability estimate for the solution of problem (4) in the arbitrary Banach space $E$. Nevertheless, in [20], the coercive stability estimate for the solution of problem (4) was established, 
when the space $E$ is replaced by the fractional spaces $E_{\alpha}(0<\alpha<1)$ which is defined above under the condition

$$
\left\|B(t) A^{-1}\right\|_{E \mapsto E} \leq \frac{1-\alpha}{M 2^{2-\alpha}}
$$

for every $t \geq 0$, where $M$ is the constant from equation (5). However, the condition (7) is stronger than (6) and $E \neq E_{\alpha}$. Finally, in papers [21, 22], theorems on the well-posedness in Hölder spaces in $t$ of the initial value problem for the delay parabolic equation

$$
\left\{\begin{array}{l}
\varepsilon \frac{d v(t)}{d t}+A v(t)=B(t) v(t-\omega)+f(t), \quad t \geq 0, \\
v(t)=g(t) \quad(-\omega \leq t \leq 0)
\end{array}\right.
$$

in an arbitrary Banach space $E$ with the small positive parameter $\varepsilon$ in the high derivative and with the unbounded linear operators $A$ and $B(t)$ in $E$ with dense domains $D(A) \subseteq$ $D(B(t))$ were established.

Additionally, using the first and second order of the accuracy implicit difference schemes for differential equations without the presence of delay, the first and second order of the accuracy implicit difference schemes,

$$
\begin{aligned}
& \left\{\begin{array}{l}
\frac{1}{\tau}\left(u_{k}-u_{k-1}\right)+A u_{k}=B_{k} u_{k-N}+\varphi_{k}, \quad \varphi_{k}=f\left(t_{k}\right), B_{k}=B\left(t_{k}\right), t_{k}=k \tau, 1 \leq k, \\
N \tau=\omega, \quad u_{k}=g\left(t_{k}\right), \quad t_{k}=k \tau,-N \leq k \leq 0,
\end{array}\right. \\
& \left\{\begin{array}{l}
\frac{1}{\tau}\left(u_{k}-u_{k-1}\right)+A S u_{k}=S B_{k} g\left(t_{k-N}-\frac{\tau}{2}\right), \quad 1 \leq k \leq N, t_{k-N}=(k-N) \tau, \\
\frac{1}{\tau}\left(u_{k}-u_{k-1}\right)+A S u_{k}=\frac{1}{2} S B_{k}\left(u_{k-N}+u_{k-N-1}\right)+\varphi_{k}, \quad \varphi_{k}=S f\left(t_{k}-\frac{\tau}{2}\right), \\
B_{k}=B\left(t_{k}-\frac{\tau}{2}\right), \quad t_{k}=k \tau, N+1 \leq k,
\end{array}\right.
\end{aligned}
$$

are presented for approximate solutions of the initial value problem (4). Here, we will put $S=I+\frac{1}{2} \tau A$.

The main aim of present paper is to study the well-posedness of the difference schemes (9) and (10). We establish the coercive stability estimates in fractional spaces $E_{\alpha}(0<\alpha<1)$ under the assumption (7). In practice, the coercive stability estimates in Hölder norms for the solutions of difference schemes for the approximate solutions of the mixed problem of delay parabolic equations are obtained.

The paper is organized as follows. In Section 2, theorems on coercive stability of difference schemes (9) and (10) are established. In Section 3, the coercive stability estimates in Hölder norms for the solutions of difference schemes for the approximate solutions of delay parabolic equations are obtained. Finally, Section 4 is our conclusion.

\section{The well-posedness of difference schemes (9) and (10)}

First, we consider the difference scheme (9) when $A^{-1}$ and $B(t)$ commute, i.e.

$$
A^{-1} B(t) u=B(t) A^{-1} u, \quad u \in D(A) .
$$


Theorem 1 Assume that the condition (7) holds for every $t \geq 0$, where $M$ is the constant from (5). Then for the solution of the difference scheme (9), the estimate

$$
\begin{aligned}
& \left\|\frac{u_{k}-u_{k-1}}{\tau}\right\|_{E_{\alpha}}+\left\|A u_{k}\right\|_{E_{\alpha}} \\
& \quad \leq \max _{-N \leq i \leq 0}\left\|A g\left(t_{i}\right)\right\|_{E_{\alpha}}+\frac{M 2^{2-\alpha}}{1-\alpha}\left[\sum_{n=1}^{\left[\frac{k}{N}\right]} \sup _{(n-1) N+1 \leq i \leq n N}\left\|\varphi_{i}\right\|_{E_{\alpha}}+\sup _{\left[\frac{k}{N}\right] N+1 \leq i \leq k}\left\|\varphi_{i}\right\|_{E_{\alpha}}\right]
\end{aligned}
$$

holds for any $k \geq 1$. Here and in future we put $\sum_{n=1}^{m} a_{n}=0$ if $m<1$.

Proof Let us consider $1 \leq k \leq N$. In this case

$$
u_{k}=R^{k} g(0)+\sum_{j=1}^{k} R^{k-j+1} B_{j} g\left(t_{j-N}\right) \tau+\sum_{j=1}^{k} R^{k-j+1} B_{j} \varphi_{j} \tau=v_{k}+w_{k},
$$

where

$$
\begin{aligned}
& v_{k}=R^{k} g(0)+\sum_{j=1}^{k} R^{k-j+1} B_{j} g\left(t_{j-N}\right) \tau, \\
& w_{k}=\sum_{j=1}^{k} R^{k-j+1} B_{j} \varphi_{j} \tau, \quad R=(1+\tau A)^{-1} .
\end{aligned}
$$

Let us estimate $A v_{k}$ and $A w_{k}$ for any $k \geq 1$. Using the formula

$$
(I+\tau A)^{-k}=\frac{1}{(k-1) !} \int_{0}^{\infty} t^{k-1} e^{-t} e^{-\tau t A} d t, \quad k \geq 1,
$$

condition (11) and estimates (5) and (7), we obtain

$$
\begin{aligned}
\lambda^{1-\alpha} \| & A e^{-\lambda A} A v_{k} \|_{E} \\
\leq & \lambda^{1-\alpha} \frac{1}{(k-1) !} \int_{0}^{\infty} t^{k-1} e^{-t}\left\|A e^{-(\tau t+\lambda) A} A g(0)\right\|_{E} d t \\
& +\lambda^{1-\alpha} \sum_{j=1}^{k} \tau \frac{1}{(k-j) !} \int_{0}^{\infty} t^{k-j} e^{-t}\left\|A e^{-\frac{(\tau t+\lambda) A}{2}}\right\|_{E \mapsto E}\left\|B_{j} A^{-1}\right\|_{E \mapsto E} \\
& \times\left\|A e^{-\frac{(\tau t+\lambda) A}{2}} A g\left(t_{j-N}\right)\right\|_{E} d t \\
\leq & \lambda^{1-\alpha} \frac{1}{(k-1) !} \int_{0}^{\infty} t^{k-1} e^{-t} \frac{d t}{(t \tau+\lambda)^{1-\alpha}}\|A g(0)\|_{E_{\alpha}} \\
& +\lambda^{1-\alpha} \frac{1-\alpha}{M 2^{2-\alpha}} \sum_{j=1}^{k} \tau \frac{1}{(k-j) !} \int_{0}^{\infty} t^{k-j} e^{-t} \frac{M 2^{2-\alpha} d t}{(t \tau+\lambda)^{2-\alpha}}\left\|A g\left(t_{j-N}\right)\right\|_{E_{\alpha}} \\
\leq & J \max _{-N \leq i \leq 0}\left\|A g\left(t_{i}\right)\right\|_{E_{\alpha}},
\end{aligned}
$$


where

$$
\begin{aligned}
J= & \lambda^{1-\alpha} \frac{1}{(k-1) !} \int_{0}^{\infty} t^{k-1} e^{-t} \frac{d t}{(t \tau+\lambda)^{1-\alpha}} \\
& +\lambda^{1-\alpha}(1-\alpha) \sum_{j=1}^{k} \tau \frac{1}{(k-j) !} \int_{0}^{\infty} t^{k-j} e^{-t} \frac{d t}{(t \tau+\lambda)^{2-\alpha}} .
\end{aligned}
$$

Making the substitution $k-j=m$ and integrating by parts, we obtain

$$
\begin{aligned}
\lambda^{1-\alpha}(1-\alpha) \sum_{m=0}^{k-1} \tau \frac{1}{m !} \int_{0}^{\infty} t^{m} e^{-t} \frac{d t}{(t \tau+\lambda)^{2-\alpha}} \\
=1-\lambda^{1-\alpha} \int_{0}^{\infty} e^{-t} \frac{d t}{(t \tau+\lambda)^{1-\alpha}}+\lambda^{1-\alpha} \int_{0}^{\infty} \sum_{m=1}^{k-1}\left[\frac{1}{(m-1) !} t^{m-1}-\frac{1}{m !} t^{m}\right] e^{-t} \frac{d t}{(t \tau+\lambda)^{1-\alpha}} \\
=1-\lambda^{1-\alpha} \int_{0}^{\infty} e^{-t} \frac{d t}{(t \tau+\lambda)^{1-\alpha}}+\lambda^{1-\alpha} \int_{0}^{\infty} e^{-t} \frac{d t}{(t \tau+\lambda)^{1-\alpha}} \\
\quad-\lambda^{1-\alpha} \frac{1}{(k-1) !} \int_{0}^{\infty} t^{k-1} e^{-t} \frac{d t}{(t \tau+\lambda)^{1-\alpha}} \\
=1-\lambda^{1-\alpha} \frac{1}{(k-1) !} \int_{0}^{\infty} t^{k-1} e^{-t} \frac{d t}{(t \tau+\lambda)^{1-\alpha}}
\end{aligned}
$$

Therefore $J=1$ and

$$
\lambda^{1-\alpha}\left\|A e^{-\lambda A} A v_{k}\right\|_{E} \leq \max _{-N \leq i \leq 0}\left\|A g\left(t_{i}\right)\right\|_{E_{\alpha}}
$$

for every $1 \leq k \leq N$ and $\lambda>0$. This shows that

$$
\left\|A v_{k}\right\|_{E_{\alpha}} \leq \max _{-N \leq i \leq 0}\left\|A g\left(t_{i}\right)\right\|_{E_{\alpha}}
$$

for every $1 \leq k \leq N$. Using formula (13), and the estimate (5), we obtain

$$
\lambda^{1-\alpha}\left\|A e^{-\lambda A} A w_{k}\right\|_{E} \leq M 2^{2-\alpha} \lambda^{1-\alpha} \sum_{j=1}^{k} \tau \frac{1}{(k-j) !} \int_{0}^{\infty} t^{k-j} e^{-t} \frac{d t}{(t \tau+\lambda)^{2-\alpha}}\left\|\varphi_{j}\right\|_{E_{\alpha}} .
$$

Applying the inequality

$$
\sum_{j=1}^{k} \frac{1}{(k-j) !} t^{k-j} \leq e^{t}
$$

we get

$$
\begin{aligned}
\lambda^{1-\alpha}\left\|A e^{-\lambda A} A w_{k}\right\|_{E} & \leq M 2^{2-\alpha} \lambda^{1-\alpha} \tau \int_{0}^{\infty} \frac{d t}{(t \tau+\lambda)^{2-\alpha}} \sup _{1 \leq j \leq k}\left\|\varphi_{j}\right\|_{E_{\alpha}} \\
& =\frac{M 2^{2-\alpha}}{1-\alpha} \sup _{1 \leq j \leq k}\left\|\varphi_{j}\right\|_{E_{\alpha}}
\end{aligned}
$$


for every $1 \leq k \leq N$ and $\lambda>0$. This shows that

$$
\left\|A w_{k}\right\|_{E_{\alpha}} \leq \frac{M 2^{2-\alpha}}{1-\alpha} \sup _{1 \leq j \leq k}\left\|\varphi_{j}\right\|_{E_{\alpha}}
$$

for every $1 \leq k \leq N$. Using the triangle inequality and the estimates (14) and (15), we get

$$
\left\|A u_{k}\right\|_{E_{\alpha}} \leq \max _{-N \leq i \leq 0}\left\|g\left(t_{i}\right)\right\|_{E_{\alpha}}+\frac{M 2^{2-\alpha}}{1-\alpha} \sup _{1 \leq j \leq k}\left\|\varphi_{j}\right\|_{E_{\alpha}}
$$

Applying mathematical induction, one can easily show that it is true for every $k$. Actually, suppose that the estimate (16) is true for $(n-1) N \leq k \leq n N, n=1,2,3, \ldots$ Letting $k=$ $m+n N$, we have

$$
\frac{1}{\tau}\left(u_{m+n N}-u_{m+n N-1}\right)+A u_{m+n N}=B_{m+n N} u_{m+n N-N}+\varphi_{m+n N}, \quad 1 \leq m \leq N .
$$

Using the estimate (16), we obtain

$$
\begin{aligned}
& \left\|A u_{k}\right\|_{E_{\alpha}} \\
& \leq \max _{1 \leq m \leq N}\left\|A u_{m+n N-N}\right\|_{E_{\alpha}}+\frac{M 2^{2-\alpha}}{1-\alpha} \sup _{\left[\frac{k}{N}\right] N+1 \leq i \leq k}\left\|\varphi_{i}\right\|_{E_{\alpha}} \\
& \leq \max _{-N \leq i \leq 0}\left\|A g\left(t_{i}\right)\right\|_{E_{\alpha}}+\frac{M 2^{2-\alpha}}{1-\alpha} \sum_{n=1}^{\left[\frac{k}{N}\right]} \sup _{(n-1) N+1 \leq i \leq n N}\left\|\varphi_{i}\right\|_{E_{\alpha}}+\frac{M 2^{2-\alpha}}{1-\alpha} \sup _{\left[\frac{k}{N}\right] N+1 \leq i \leq k}\left\|\varphi_{i}\right\|_{E_{\alpha}} \\
& \quad \leq \max _{-N \leq i \leq 0}\left\|A g\left(t_{i}\right)\right\|_{E_{\alpha}}+\frac{M 2^{2-\alpha}}{1-\alpha}\left[\sum_{n=1}^{\left[\frac{k}{N}\right]} \sup _{(n-1) N+1 \leq i \leq n N}\left\|\varphi_{i}\right\|_{E_{\alpha}}+\sup _{\left[\frac{k}{N}\right] N+1 \leq i \leq k}\left\|\varphi_{i}\right\|_{E_{\alpha}}\right]
\end{aligned}
$$

for every $n N+1 \leq k \leq(n+1) N, n=1,2,3, \ldots$ Theorem 1 is proved.

Now, we consider the difference scheme (9) when

$$
A^{-1} B(t) x \neq B(t) A^{-1} x, \quad x \in D(A)
$$

for some $t \geq 0$.

Recall that (see, for example, [23, Chapter 2, p.116]) $A$ is a strongly positive operator in a Banach space $E$ iff its spectrum $\sigma(A)$ lies in the interior of the sector of the angle $\varphi, 0<2 \varphi<\pi$, symmetric with respect to the real axis, and if on the edges of this sector, $S_{1}=\left[z=\rho e^{i \varphi}: 0 \leq \rho<\infty\right]$ and $S_{2}=\left[z=\rho e^{-i \varphi}: 0 \leq \rho<\infty\right]$, and outside it the resolvent $(z-A)^{-1}$ is subject to the bound

$$
\left\|(z-A)^{-1}\right\|_{E \rightarrow E} \leq \frac{M_{1}}{1+|z|}
$$

for some $M_{1}>0$. First of all let us give lemmas from the paper [12] that will be needed in the sequel. 
Lemma 1 For any $z$ on the edges of the sector,

$$
S_{1}=\left[z=\rho e^{i \varphi}: 0 \leq \rho<\infty\right] \text { and } S_{2}=\left[z=\rho e^{-i \varphi}: 0 \leq \rho<\infty\right]
$$

and outside it the estimate

$$
\left\|A(z-A)^{-1} x\right\|_{E} \leq \frac{M_{1}^{\alpha} M^{\alpha}\left(1+M_{1}\right)^{1-\alpha} 2^{(2-\alpha) \alpha}}{\alpha(1-\alpha)(1+|z|)^{\alpha}}\|x\|_{E_{\alpha}}
$$

holds for any $x \in E_{\alpha}$. Here and in the future $M$ and $M_{1}$ are the same constants as of the estimates (5) and (17).

Lemma 2 Let for all $s \geq 0$ the operator $B(s) A^{-1}-A^{-1} B(s)$ with domain which coincides with $D(A)$ permit the closure $\overline{B(s) A^{-1}-A^{-1} B(s)}$ bounded in $E$. Then for all $\tau>0$ the following estimate holds:

$$
\begin{aligned}
& \left\|A^{-1}\left[A e^{-\tau A} B(s)-B(s) A e^{-\tau A}\right] x\right\|_{E} \\
& \quad \leq \frac{e(\alpha+1) M^{\alpha} M_{1}^{1+\alpha}\left(1+2 M_{1}\right)\left(1+M_{1}\right)^{1-\alpha} 2^{(2-\alpha) \alpha}\|Q\|_{E \mapsto E}\|x\|_{E_{\alpha}}}{\tau^{1-\alpha} \pi \alpha^{2}(1-\alpha)} .
\end{aligned}
$$

Here $Q=\overline{A^{-1}(A B(s)-B(s) A) A^{-1}}$.

Suppose that

$$
\begin{aligned}
& \overline{\left\|A^{-1}(A B(t)-B(t) A) A^{-1}\right\|_{E \mapsto E}} \\
& \leq \frac{\pi(1-\alpha)^{2} \alpha^{2} \varepsilon}{e M^{1+\alpha} M_{1}^{1+\alpha}\left(1+2 M_{1}\right)\left(1+M_{1}\right)^{1-\alpha} 2^{2+\alpha-\alpha^{2}}(1+\alpha)}
\end{aligned}
$$

holds for every $t \geq 0$. Here and in the future $\varepsilon$ is a constant, $0 \leq \varepsilon \leq 1$.

The use of Lemmas 1 and 2 enables us to establish the following statement.

Theorem 2 Assume that the condition

$$
{\overline{\left\|A^{-1} B(t)\right\|_{E \mapsto E}}} \frac{(1-\alpha)(1-\varepsilon)}{M 2^{2-\alpha}}
$$

holds for every $t \geq 0$. Then for the solution of the difference scheme (9), the coercive estimate (12) holds.

Proof Let us consider $1 \leq k \leq N$. Using formula (13), we can write

$$
\begin{aligned}
& \lambda^{1-\alpha} A e^{-\lambda A} A v_{k} \\
& =\lambda^{1-\alpha} \frac{1}{(k-1) !} \int_{0}^{\infty} t^{k-1} e^{-t} A e^{-(\tau t+\lambda) A} A g(0) d t \\
& \quad+\lambda^{1-\alpha} \sum_{j=1}^{k} \tau \frac{1}{(k-j) !} \int_{0}^{\infty} t^{k-j} e^{-t} e^{-\frac{\tau t+\lambda}{2} A} B_{j} A e^{-\frac{\tau t+\lambda}{2} A} A g\left(t_{j-N}\right) d t
\end{aligned}
$$




$$
\begin{aligned}
& +\lambda^{1-\alpha} \sum_{j=1}^{k} \tau \frac{1}{(k-j) !} \int_{0}^{\infty} t^{k-j} e^{-t} A e^{-\frac{\tau t+\lambda}{2} A} A^{-1}\left[e^{-\frac{\tau t+\lambda}{2} A} A B_{j}-B_{j} A e^{-\frac{\tau t+\lambda}{2} A}\right] A g\left(t_{j-N}\right) d t \\
= & T_{1}+T_{2}+T_{3} .
\end{aligned}
$$

Using the estimates (5), (17), and condition (19), we obtain

$$
\begin{aligned}
\left\|T_{1}\right\|_{E} \leq & \lambda^{1-\alpha} \frac{1}{(k-1) !} \int_{0}^{\infty} t^{k-1} e^{-t}\left\|A e^{-(\tau t+\lambda) A} A g(0)\right\|_{E} d t \\
\leq & \lambda^{1-\alpha} \frac{1}{(k-1) !} \int_{0}^{\infty} t^{k-1} e^{-t} \frac{d t}{(t \tau+\lambda)^{1-\alpha}} \max _{-N \leq i \leq 0}\left\|A g\left(t_{i}\right)\right\|_{E_{\alpha}}, \\
\left\|T_{2}\right\|_{E} \leq & \lambda^{1-\alpha} \sum_{j=1}^{k} \tau \frac{1}{(k-j) !} \int_{0}^{\infty} t^{k-j} e^{-t}\left\|A e^{-\frac{\tau t+\lambda}{2} A}\right\|_{E \rightarrow E}\left\|A^{-1} B_{j}\right\|_{E \rightarrow E} \\
& \times\left\|A e^{-\frac{\tau t+\lambda}{2} A} A g\left(t_{j-N}\right)\right\|_{E} d t \\
\leq & \frac{\lambda^{1-\alpha}(1-\alpha)(1-\varepsilon)}{M 2^{2-\alpha}} \sum_{j=1}^{k} \tau \frac{1}{(k-j) !} \int_{0}^{\infty} t^{k-j} e^{-t} \frac{M 2^{2-\alpha} d t}{(t \tau+\lambda)^{2-\alpha}}\left\|A g\left(t_{j-N}\right)\right\|_{E_{\alpha}} \\
\leq & \lambda^{1-\alpha}(1-\alpha)(1-\varepsilon) \sum_{j=1}^{k} \tau \frac{1}{(k-j) !} \int_{0}^{\infty} t^{k-j} e^{-t} \frac{d t}{(t \tau+\lambda)^{2-\alpha}} \max _{-N \leq i \leq 0}\left\|A g\left(t_{i}\right)\right\|_{E_{\alpha}}
\end{aligned}
$$

for every $1 \leq k \leq N$ and $\lambda>0$. Now let us estimate $T_{3}$. By Lemma 2 and using the estimate (20), we can obtain

$$
\begin{aligned}
\left\|T_{3}\right\|_{E} \leq & \lambda^{1-\alpha} \sum_{j=1}^{k} \tau \frac{1}{(k-j) !} \int_{0}^{\infty} t^{k-j} e^{-t}\left\|A e^{-\frac{\tau t+\lambda}{2} A}\right\|_{E \rightarrow E} \\
& \times\left\|A^{-1}\left[A e^{-\frac{\tau t+\lambda}{2} A} B_{j}-B_{j} A e^{-\frac{\tau t+\lambda}{2} A}\right] A g\left(t_{j-N}\right)\right\|_{E} d t \\
\leq & \lambda^{1-\alpha} \max _{1 \leq k \leq N}\left\|\overline{A^{-1}\left(A B_{j}-B_{j} A\right) A^{-1}}\right\|_{E \rightarrow E} \sum_{j=1}^{k} \tau \frac{1}{(k-j) !} \\
& \times \int_{0}^{\infty} t^{k-j} e^{-t} \frac{e(1+\alpha) M^{\alpha} M_{1}^{1+\alpha}\left(1+2 M_{1}\right)\left(1+M_{1}\right)^{1-\alpha} 2^{(2-\alpha) \alpha}}{\pi \alpha^{2}(1-\alpha)} \\
& \times \frac{M 2^{2-\alpha} d t}{(t \tau+\lambda)^{2-\alpha}}\left\|A g\left(t_{j-N}\right)\right\|_{E_{\alpha}} \\
\leq & \lambda^{1-\alpha}(1-\alpha) \varepsilon \sum_{j=1}^{k} \tau \frac{1}{(k-j) !} \int_{0}^{\infty} t^{k-j} e^{-t} \frac{d t}{(t \tau+\lambda)^{2-\alpha}} \max _{-N \leq i \leq 0}\left\|A g\left(t_{i}\right)\right\|_{E_{\alpha}}
\end{aligned}
$$

for every $1 \leq k \leq N$ and $\lambda>0$. Using the triangle inequality, we obtain

$$
\lambda^{1-\alpha}\left\|A e^{-\lambda A} A v_{k}\right\|_{E} \leq \max _{-N \leq i \leq 0} J\left\|A g\left(t_{i}\right)\right\|_{E_{\alpha}}
$$

for every $1 \leq k \leq N$ and $\lambda>0$. This shows that

$$
\left\|A v_{k}\right\|_{E_{\alpha}} \leq \max _{-N \leq i \leq 0}\left\|A g\left(t_{i}\right)\right\|_{E_{\alpha}}
$$


for every $1 \leq k \leq N$. Using the triangle inequality and the last estimate and (15), we get

$$
\left\|A u_{k}\right\|_{E_{\alpha}} \leq \max _{-N \leq i \leq 0}\left\|A g\left(t_{i}\right)\right\|_{E_{\alpha}}+\frac{M 2^{2-\alpha}}{1-\alpha} \sup _{1 \leq i \leq k}\left\|\varphi_{i}\right\|_{E_{\alpha}}
$$

In a similar manner as Theorem 1, applying mathematical induction, one can easily show that it is true for every $k$. Theorem 2 is proved.

Now we consider the difference scheme (10). We have not been able to obtain the same result for the solution of the difference scheme (10) in spaces $E_{\alpha}$ under assumption (7). Nevertheless, for the solution of the difference scheme (10) the coercive stability estimate in the norm of same fractional spaces $E_{\alpha}(0<\alpha<1)$ under the supplementary restriction of the operator $A$ is established.

Theorem 3 Suppose that the following estimates hold:

$$
\begin{aligned}
& \left\|(I+\tau A)(I+\tau A S)^{-1}\right\|_{E \mapsto E} \leq 1, \\
& \left\|S(I+\tau A)(I+\tau A S)^{-1}\right\|_{E \mapsto E} \leq \frac{1+\sqrt{2}}{2},
\end{aligned}
$$

and

$$
\left\|B(t) A^{-1}\right\|_{E \mapsto E} \leq \frac{(1-\alpha)}{M 2^{1-\alpha}(1+\sqrt{2})}, \quad t \geq 0 .
$$

Then for the solution of the difference scheme (10), the coercive estimate (12) holds.

Proof Let us consider $1 \leq k \leq N$. In this case

$$
\begin{aligned}
u_{k} & =R^{k} g(0)+\sum_{j=1}^{k} R^{k-j+1}\left(I+\frac{\tau A}{2}\right) B_{j}\left(g\left(t_{j-N}\right)+g\left(t_{j-N-1}\right)\right) \tau+\sum_{j=1}^{k} R^{k-j+1}\left(I+\frac{\tau A}{2}\right) \varphi_{j} \tau \\
& =v_{k}+w_{k}
\end{aligned}
$$

where

$$
\begin{aligned}
& v_{k}=R^{k} g(0)+\sum_{j=1}^{k} R^{k-j+1}\left(I+\frac{\tau A}{2}\right) B_{j}\left(g\left(t_{j-N}\right)+g\left(t_{j-N-1}\right)\right) \tau, \\
& w_{k}=\sum_{j=1}^{k} R^{k-j+1}\left(I+\frac{\tau A}{2}\right) \varphi_{j} \tau, \quad R=\left(I+\tau A+\frac{(\tau A)^{2}}{2}\right)^{-1} .
\end{aligned}
$$

Let us estimate $A v_{k}$ and $A w_{k}$ for any $k \geq 1$. Using formula (22), condition (11), and the estimates (5) and (7), we obtain

$$
\begin{aligned}
& \lambda^{1-\alpha}\left\|A e^{-\lambda A} A v_{k}\right\|_{E} \\
& \quad \leq\left\|\left((I+\tau A)\left(I+\tau A+\frac{(\tau A)^{2}}{2}\right)^{-1}\right)^{k}\right\|_{E \mapsto E}
\end{aligned}
$$




$$
\begin{aligned}
& \times \lambda^{1-\alpha} \frac{1}{(k-1) !} \int_{0}^{\infty} t^{k-1} e^{-t}\left\|A e^{-(\tau t+\lambda) A} A g(0)\right\|_{E} d t \\
& +\lambda^{1-\alpha} \sum_{j=1}^{k} \tau\left\|\left((I+\tau A)\left(I+\tau A+\frac{(\tau A)^{2}}{2}\right)^{-1}\right)^{k-j}\right\|_{E \mapsto E} \\
& \times\left\|\left(I+\frac{\tau A}{2}\right)(I+\tau A)\left(I+\tau A+\frac{(\tau A)^{2}}{2}\right)^{-1}\right\|_{E \mapsto E} \\
& \times \frac{1}{(k-j) !} \int_{0}^{\infty} t^{k-j} e^{-t}\left\|A e^{-\frac{\tau t+\lambda}{2} A}\right\|_{E \rightarrow E}\left\|B_{j} A^{-1}\right\|_{E \rightarrow E} \\
& \times\left\|A e^{-\frac{\tau t+\lambda}{2} A} \frac{1}{2} A\left(g\left(t_{j-N}\right)+g\left(t_{j-N-1}\right)\right)\right\|_{E} d t \\
& \leq \lambda^{1-\alpha} \frac{1}{(k-1) !} \int_{0}^{\infty} t^{k-1} e^{-t} \frac{d t}{(t \tau+\lambda)^{1-\alpha}}\|A g(0)\|_{E_{\alpha}}+\frac{(1+\sqrt{2})}{2} \frac{1-\alpha}{M 2^{1-\alpha}(1+\sqrt{2})} \\
& \quad \times \sum_{j=1}^{k} \frac{1}{(k-j) !} \int_{0}^{\infty} t^{k-j} e^{-t} \frac{M 2^{2-\alpha} d t}{(t \tau+\lambda)^{2-\alpha}}\left\|\frac{1}{2} A\left(g\left(t_{j-N}\right)+g\left(t_{j-N-1}\right)\right)\right\|_{E_{\alpha}} \\
& \leq J \max _{-N \leq i \leq 0}\left\|A g\left(t_{i}\right)\right\|_{E_{\alpha}} \leq \max _{-N \leq i \leq 0}\left\|A g\left(t_{i}\right)\right\|_{E_{\alpha}}
\end{aligned}
$$

for every $1 \leq k \leq N$ and $\lambda>0$. This shows that

$$
\left\|A v_{k}\right\|_{E_{\alpha}} \leq \max _{-N \leq i \leq 0}\left\|A g\left(t_{i}\right)\right\|_{E_{\alpha}}
$$

for every $1 \leq k \leq N$. Using formula (22), the condition (21), and the estimate (5), we obtain

$$
\begin{aligned}
\lambda^{1-\alpha}\left\|A e^{-\lambda A} w_{k}\right\|_{E} \leq & \lambda^{1-\alpha} \sum_{j=1}^{k} \tau\left\|\left((I+\tau A)\left(I+\tau A+\frac{(\tau A)^{2}}{2}\right)^{-1}\right)^{k-j}\right\|_{E \mapsto E} \\
& \times\left\|\left(I+\frac{\tau A}{2}\right)(I+\tau A)\left(I+\tau A+\frac{(\tau A)^{2}}{2}\right)^{-1}\right\|_{E \mapsto E} \\
& \times \frac{1}{(k-j) !} \int_{0}^{\infty} t^{k-j} e^{-t} \frac{d t}{(t \tau+\lambda)^{1-\alpha}}\left\|\varphi_{j}\right\|_{E_{\alpha}} \\
\leq & M 2^{2-\alpha} \lambda^{1-\alpha} \sum_{j=1}^{k} \tau \frac{1}{(k-j) !} \int_{0}^{\infty} t^{k-j} e^{-t} \frac{d t}{(t \tau+\lambda)^{2-\alpha}}\left\|\varphi_{j}\right\|_{E_{\alpha}} \\
\leq & \frac{M 2^{2-\alpha}}{1-\alpha} \sup _{1 \leq j \leq k}\left\|\varphi_{j}\right\|_{E_{\alpha}}
\end{aligned}
$$

for every $1 \leq k \leq N$ and $\lambda>0$. This shows that

$$
\left\|A w_{k}\right\|_{E_{\alpha}} \leq \frac{M 2^{2-\alpha}}{1-\alpha} \sup _{1 \leq j \leq k}\left\|\varphi_{j}\right\|_{E_{\alpha}}
$$

for every $1 \leq k \leq N$. Using the triangle inequality and the estimates (23) and (24), we get

$$
\left\|A u_{k}\right\|_{E_{\alpha}} \leq \max _{-N \leq i \leq 0}\left\|g\left(t_{i}\right)\right\|_{E_{\alpha}}+\frac{M 2^{2-\alpha}}{1-\alpha} \sup _{1 \leq j \leq k}\left\|\varphi_{j}\right\|_{E_{\alpha}} .
$$


In a similar manner as Theorem 1, applying mathematical induction, one can easily show that it is true for every $k$. Theorem 3 is established.

Now, we consider the difference scheme (10) when

$$
A^{-1} B(t) x \neq B(t) A^{-1} x, \quad x \in D(A)
$$

for some $t \geq 0$. Suppose that the operator $B(t) A^{-1}-A^{-1} B(t)$, with domain which coincides with $D(A)$, permits the closure bounded in $E$ and that the estimate

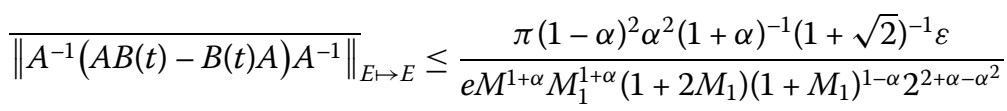

holds for every $t \geq 0$ and some $\varepsilon \in[0,1]$.

Theorem 4 Assume that all conditions of Theorem 2 and Theorem 3 are satisfied. Then for the solution of the difference scheme (10), the estimate (12) holds.

Proof Let us consider $1 \leq k \leq N$. Using formula (22), the estimates (5), (17), and the condition (19), we obtain

$$
\begin{aligned}
& \lambda^{1-\alpha} A e^{-\lambda A} A v_{k} \\
& =\left((I+\tau A)\left(I+\tau A+\frac{(\tau A)^{2}}{2}\right)^{-1}\right)^{k} \lambda^{1-\alpha} \frac{1}{(k-1) !} \int_{0}^{\infty} t^{k-1} e^{-t} A e^{-(\tau t+\lambda) A} A g(0) d t \\
& +\lambda^{1-\alpha} \sum_{j=1}^{k} \tau\left((I+\tau A)\left(I+\tau A+\frac{(\tau A)^{2}}{2}\right)^{-1}\right)^{k-j}\left(I+\frac{\tau A}{2}\right)(I+\tau A) \\
& \times\left(I+\tau A+\frac{(\tau A)^{2}}{2}\right)^{-1} \frac{1}{(k-j) !} \int_{0}^{\infty} t^{k-j} e^{-t} e^{-\frac{(\tau t+\lambda) A}{2}} B_{j} A e^{-\frac{(\tau t+\lambda) A}{2}} \\
& \times \frac{1}{2} A\left(g\left(t_{j-N}\right)+g\left(t_{j-N-1}\right)\right) d t \\
& +\lambda^{1-\alpha} \sum_{j=1}^{k} \tau\left((I+\tau A)\left(I+\tau A+\frac{(\tau A)^{2}}{2}\right)^{-1}\right)^{k-j}\left(I+\frac{\tau A}{2}\right)(I+\tau A) \\
& \times\left(I+\tau A+\frac{(\tau A)^{2}}{2}\right)^{-1} \frac{1}{(k-j) !} \int_{0}^{\infty} t^{k-j} e^{-t} A e^{-\frac{(\tau t+\lambda) A}{2}} A^{-1} \\
& \times\left[e^{-\frac{(\tau t+\lambda) A}{2}} A B_{j}-B_{j} A e^{-\frac{(\tau t+\lambda) A}{2}}\right] \frac{1}{2} A\left(g\left(t_{j-N}\right)+g\left(t_{j-N-1}\right)\right) d t \\
& =P_{1}+P_{2}+P_{3} \text {. }
\end{aligned}
$$

Using the estimates (5), (17), and the condition (19), we obtain

$$
\begin{aligned}
\left\|P_{1}\right\|_{E} \leq & \left\|\left((I+\tau A)\left(I+\tau A+\frac{(\tau A)^{2}}{2}\right)^{-1}\right)^{k}\right\|_{E \mapsto E} \\
& \times \lambda^{1-\alpha} \frac{1}{(k-1) !} \int_{0}^{\infty} t^{k-1} e^{-t}\left\|A e^{-(\tau t+\lambda) A} A g(0)\right\|_{E} d t
\end{aligned}
$$




$$
\begin{aligned}
\leq & \lambda^{1-\alpha} \frac{1}{(k-1) !} \int_{0}^{\infty} t^{k-1} e^{-t} \frac{d t}{(t \tau+\lambda)^{1-\alpha}} \max _{-N \leq i \leq 0}\left\|A g\left(t_{i}\right)\right\|_{E_{\alpha}}, \\
\left\|P_{2}\right\|_{E} \leq & \lambda^{1-\alpha} \sum_{j=1}^{k} \tau\left\|\left((I+\tau A)\left(I+\tau A+\frac{(\tau A)^{2}}{2}\right)^{-1}\right)^{k-j}\right\|_{E \mapsto E} \\
& \times\left\|\left(I+\frac{\tau A}{2}\right)(I+\tau A)\left(I+\tau A+\frac{(\tau A)^{2}}{2}\right)^{-1}\right\| \\
& \times \frac{1}{(k-j) !} \int_{0}^{\infty} t^{k-j} e^{-t}\left\|A e^{-\frac{(\tau t+\lambda) A}{2}}\right\|_{E \rightarrow E}\left\|\overline{A^{-1} B_{j}}\right\|_{E \rightarrow E} \\
& \times\left\|A e^{-\frac{(\tau t+\lambda) A}{2}} \frac{1}{2} A\left(g\left(t_{j-N}\right)+g\left(t_{j-N-1}\right)\right)\right\|_{E} d t \\
\leq & \frac{(1+\sqrt{2})}{2} \frac{\lambda^{1-\alpha}(1-\alpha)(1-\varepsilon)}{M 2^{1-\alpha}(1+\sqrt{2})} \\
& \times \sum_{j=1}^{k} \tau \frac{1}{(k-j) !} \int_{0}^{\infty} t^{k-j} e^{-t} \frac{M 2^{2-\alpha} d t}{(t \tau+\lambda)^{2-\alpha}}\left\|\frac{1}{2} A\left(g\left(t_{j-N}\right)+g\left(t_{j-N-1}\right)\right)\right\|_{E_{\alpha}} \\
\leq & (1-\alpha)(1-\varepsilon) \sum_{j=1}^{k} \tau \frac{1}{(k-j) !} \int_{0}^{\infty} t^{k-j} e^{-t} \frac{\lambda^{1-\alpha} d t}{(t \tau+\lambda)^{2-\alpha}} \max _{-N \leq i \leq 0}\left\|A g\left(t_{i}\right)\right\|_{E_{\alpha}}
\end{aligned}
$$

for every $1 \leq k \leq N$ and $\lambda>0$. Now let us estimate $P_{3}$. By Lemma 2 and using the estimate (20), we obtain

$$
\begin{aligned}
\left\|P_{3}\right\|_{E} \leq & \lambda^{1-\alpha} \sum_{j=1}^{k} \tau\left\|\left((I+\tau A)\left(I+\tau A+\frac{(\tau A)^{2}}{2}\right)^{-1}\right)^{k-j}\right\|_{E \mapsto E} \\
& \times\left\|\left(I+\frac{\tau A}{2}\right)(I+\tau A)\left(I+\tau A+\frac{(\tau A)^{2}}{2}\right)^{-1}\right\|_{E \mapsto E} \\
& \times \frac{1}{(k-j) !} \int_{0}^{\infty} t^{k-j} e^{-t}\left\|A e^{-\frac{(\tau t+\lambda) A}{2}}\right\|_{E \rightarrow E} \\
& \times\left\|A^{-1}\left[A e^{-\frac{(\tau t+\lambda) A}{2}} B_{j}-B_{j} A e^{-\frac{(\tau t+\lambda) A}{2}}\right] \frac{1}{2} A\left(g\left(t_{j-N}\right)+g\left(t_{j-N-1}\right)\right)\right\|_{E} d t \\
\leq & \frac{\lambda^{1-\alpha}(1+\sqrt{2})}{2} \max _{1 \leq j \leq N}\left\|\frac{A^{-1}\left(A B_{j}-B_{j} A\right) A^{-1}}{}\right\|_{E \rightarrow E} \sum_{j=1}^{k} \tau \frac{1}{(k-j) !} \\
& \times \int_{0}^{\infty} t^{k-j} e^{-t} \frac{e(1+\alpha) M^{\alpha} M_{1}^{1+\alpha}\left(1+2 M_{1}\right)\left(1+M_{1}\right)^{1-\alpha} 2^{(2-\alpha) \alpha}}{\pi \alpha^{2}(1-\alpha)} \\
& \times \frac{M 2^{2-\alpha} d t}{(t \tau+\lambda)^{2-\alpha}} \frac{1}{2}\left\|A g\left(t_{j-N}\right)+A g\left(t_{j-N-1}\right)\right\|_{E_{\alpha}} \\
\leq & \lambda^{1-\alpha}(1-\alpha) \varepsilon \sum_{j=1}^{k} \tau \frac{1}{(k-j) !} \int_{0}^{\infty} t^{k-j} e^{-t} \frac{d t}{(t \tau+\lambda)^{2-\alpha}} \max _{-N \leq i \leq 0}\left\|A g\left(t_{i}\right)\right\|_{E_{\alpha}}
\end{aligned}
$$

for every $1 \leq k \leq N$ and $\lambda>0$. Using the triangle inequality, we obtain

$$
\lambda^{1-\alpha}\left\|A e^{-\lambda A} A v_{k}\right\|_{E} \leq J \max _{-N \leq k \leq 0}\left\|A g\left(t_{k}\right)\right\|_{E_{\alpha}}
$$


for every $1 \leq k \leq N$ and $\lambda>0$. This shows that

$$
\left\|A v_{k}\right\|_{E_{\alpha}} \leq \max _{-N \leq k \leq 0}\left\|A g\left(t_{k}\right)\right\|_{E_{\alpha}}
$$

for every $1 \leq k \leq N$. Using the triangle inequality and the estimates (26) and (24), we get

$$
\left\|A u_{k}\right\|_{E_{\alpha}} \leq \max _{-N \leq i \leq 0}\left\|g\left(t_{i}\right)\right\|_{E_{\alpha}}+\frac{M 2^{2-\alpha}}{1-\alpha} \sup _{1 \leq j \leq k}\left\|\varphi_{j}\right\|_{E_{\alpha}}
$$

In a similar manner as Theorem 1, applying mathematical induction, one can easily show that it is true for every $k$. Theorem 4 is established.

Note that these abstract results are applicable to the study of the coercive stability of various delay parabolic equations with local and nonlocal boundary conditions with respect to the space variables. However, it is important to study structure of $E_{\alpha}$ for space operators in Banach spaces. The structure of $E_{\alpha}$ for some space differential and difference operators in Banach spaces has been investigated in some papers [23-32]. In Section 3, applications of Theorem 1 to the study of the coercive stability of the difference schemes for delay parabolic equations are given.

\section{Applications}

First, the initial-boundary value problem for one-dimensional delay parabolic equations is considered:

$$
\left\{\begin{array}{l}
\frac{\partial u(t, x)}{\partial t}-a(x) \frac{\partial^{2} u(t, x)}{\partial x^{2}}+\delta u(t, x) \\
\quad=b(t)\left(-a(x) \frac{\partial^{2} u(t-\omega, x)}{\partial x^{2}}+\delta u(t-\omega, x)\right)+f(t, x), \quad 0<t<\infty, x \in(0, l), \\
u(t, x)=g(t, x), \quad-\omega \leq t \leq 0, x \in[0, l], \\
u(t, 0)=u(t, l)=0, \quad-\omega \leq t<\infty
\end{array}\right.
$$

where $a(x), b(t), g(t, x), f(t, x)$ are given sufficiently smooth functions and $\delta>0$ is a sufficiently large number. It will be assumed that $a(x) \geq a>0$. The discretization of problem (27) is carried out in two steps. In the first step, the uniform grid space

$$
[0, l]_{h}=\left\{x: x_{r}=r h, 0 \leq r \leq K, K h=l\right\}
$$

is defined. To formulate the result, one needs to introduce the Banach space $\dot{C}_{h}^{\beta}=$ $C^{\beta}\left([0, l]_{h}\right)(0<\beta<1)$ of the grid functions $\varphi^{h}(x)=\left\{\varphi_{r}\right\}_{1}^{K-1}$ defined on $[0, l]_{h}$ satisfying the conditions $\varphi_{0}=\varphi_{K}=0$, equipped with the norm

$$
\left\|\varphi^{h}\right\|_{\dot{C}_{h}^{\beta}}=\left\|\varphi^{h}\right\|_{C_{h}}+\sup _{1 \leq k<k+\tau \leq K-1} \frac{\left|\varphi_{k+r}-\varphi_{k}\right|}{\tau^{\beta}} .
$$

Here and in the future, $C_{h}=C\left([0, l]_{h}\right)$ is the space of the grid functions $\varphi^{h}(x)=\left\{\varphi_{r}\right\}_{0}^{K}$ defined on $[0, l]_{h}$, equipped with the norm

$$
\left\|\varphi^{h}\right\|_{C_{h}}=\max _{0 \leq k \leq K}\left|\varphi_{k}\right|
$$


To the differential operator $A^{x}$ generated by problem (27), we assign the difference operator $A_{h}^{x}$ by the formula

$$
A_{h}^{x} \varphi^{h}(x)=\left\{-\left(a(x) \varphi_{\bar{x}}\right)_{x, r}+\delta \varphi_{r}\right\}_{1}^{K-1},
$$

acting in the space of grid functions $\varphi^{h}(x)=\left\{\varphi_{r}\right\}_{0}^{K}$ satisfying the conditions $\varphi_{0}=\varphi_{K}=0$. With the help of $A_{h}^{x}$, we arrive at the initial-boundary value problem

$$
\left\{\begin{array}{l}
\frac{d u^{h}(t, x)}{d t}+A_{h}^{x} u^{h}(t, x)=b(t) A_{h}^{x} u^{h}(t-\omega, x)+f^{h}(t, x), \quad t \geq 0, x \in[0, l]_{h}, \\
u^{h}(t, x)=g^{h}(t, x)=g(t, x) \quad(-\omega \leq t \leq 0), x \in[0, l]_{h}
\end{array}\right.
$$

for the system of ordinary differential equations. In the second step, problem (28) is replaced by the first-order accuracy in the difference scheme in $t$,

$$
\left\{\begin{array}{l}
\frac{1}{\tau}\left(u_{k}^{h}(x)-u_{k-1}^{h}(x)\right)+A_{h}^{x} u_{k}^{h}(x)=b\left(t_{k}\right) A_{h}^{x} u_{k-N}^{h}(x)+f_{k}^{h}(x), \\
f_{k}^{h}(x)=f^{h}\left(t_{k}, x\right), \quad t_{k}=k \tau, 1 \leq k, N \tau=\omega, x \in[0, l]_{h}, \\
u_{k}^{h}(x)=g^{h}\left(t_{k}, x\right), \quad t_{k}=k \tau,-N \leq k \leq 0, x \in[0, l]_{h} .
\end{array}\right.
$$

\section{Theorem 5 Assume that}

$$
\sup _{0 \leq t<\infty}|b(t)| \leq \frac{1-\alpha}{M 2^{2-\alpha}} .
$$

Then for the solution of the difference scheme (29) the following coercive stability estimates hold:

$$
\begin{aligned}
& \left\|\tau^{-1}\left(u_{k}^{h}-u_{k-1}^{h}\right)\right\|_{\dot{C}^{2 \alpha}[0, l]_{h}}+h^{-2}\left\|\Delta_{1-} \Delta_{1+} u_{k}^{h}\right\|_{\dot{C}^{2 \alpha}[0, l]_{h}} \\
& \leq M_{3}(\alpha)\left[\max _{-N \leq i \leq 0} h^{-2}\left\|\Delta_{1-} \Delta_{1+} g_{i}^{h}\right\|_{\dot{C}^{2 \alpha}[0,]_{h}}\right. \\
& \left.\quad+\sum_{n=1}^{\left[\frac{k}{N}\right]} \sup _{(n-1) N+1 \leq i \leq n N}\left\|f_{i}^{h}\right\|_{\dot{C}^{2 \alpha}[0, l]_{h}}+\sup _{\left[\frac{k}{N}\right] N+1 \leq i \leq k}\left\|f_{i}^{h}\right\|_{\dot{C}^{2 \alpha}[0, l]_{h}}\right], \\
& 0<\alpha<\frac{1}{2}
\end{aligned}
$$

for all $k \geq 1$, where $M_{3}(\alpha)$ does not depend on $g_{k}^{h}$ and $f_{k}^{h}$. Here and in the future we put

$$
\Delta_{1 \pm} \varphi^{h}(x)= \pm\left[\varphi^{h}(x \pm h)-\varphi^{h}(x)\right], \quad x, x \pm h \in[0, l]_{h} .
$$

The proof of Theorem 5 is based on the estimate

$$
\left\|e^{-t_{k} A_{h}^{x}}\right\|_{C_{h} \rightarrow C_{h}} \leq M, \quad k \geq 0
$$

and on the abstract Theorem 1, the positivity of the operator $A_{h}^{x}$ in $\dot{C}_{h}^{\beta}$, and on the following theorem on the structure of the fractional space $E_{\alpha}\left(C_{h}, A_{h}^{x}\right)$. 
Theorem 6 For any $0<\alpha<\frac{1}{2}$ the norms in the spaces $E_{\alpha}\left(C_{h}, A_{h}^{x}\right)$ and $\dot{C}_{h}^{2 \alpha}$ are equivalent uniformly in $h$ [25].

Second, the initial nonlocal boundary value problem for one-dimensional delay parabolic equations is considered:

$$
\left\{\begin{array}{l}
\frac{\partial u(t, x)}{\partial t}-a(x) \frac{\partial^{2} u(t, x)}{\partial x^{2}}+\delta u(t, x) \\
\quad=b(t)\left(-a(x) \frac{\partial^{2} u(t-\omega, x)}{\partial x^{2}}+\delta u(t-\omega, x)\right)+f(t, x), \quad 0<t<\infty, x \in(0, l), \\
u(t, x)=g(t, x), \quad-\omega \leq t \leq 0, x \in[0, l], \\
u(t, 0)=u(t, l), \quad u_{x}(t, 0)=u_{x}(t, l), \quad-\omega \leq t<\infty,
\end{array}\right.
$$

where $a(x), b(t), g(t, x), f(t, x)$ are given sufficiently smooth functions and $\delta>0$ is a sufficiently large number. It will be assumed that $a(x) \geq a>0$. The discretization of problem (32) is carried out in two steps. In the first step, let us use the discretization in the space variable $x$. To formulate the result, one needs to introduce the Banach space $C_{h}^{\beta}=C^{\beta}\left([0, l]_{h}\right)(0<\beta<1)$ of the grid functions $\varphi^{h}(x)=\left\{\varphi_{r}\right\}_{0}^{K}$ defined on $[0, l]_{h}$ satisfying the conditions $\varphi_{0}=\varphi_{K}, \varphi_{1}-\varphi_{0}=\varphi_{K}-\varphi_{K-1}$ equipped with the norm

$$
\left\|\varphi^{h}\right\|_{C_{h}^{\beta}}=\left\|\varphi^{h}\right\|_{C_{h}}+\sup _{0 \leq k<k+\tau \leq K} \frac{\left|\varphi_{k+r}-\varphi_{k}\right|}{\tau^{\beta}} .
$$

To the differential operator $A^{x}$ generated by problem (32) we assign the difference operator $\mathbb{A}_{h}^{x}$ by the formula

$$
\mathbb{A}_{h}^{x} \varphi^{h}(x)=\left\{-\left(a(x) \varphi_{\bar{x}}\right)_{x, r}+\delta \varphi_{r}\right\}_{1}^{K-1}
$$

acting in the space of grid functions $\varphi^{h}(x)=\left\{\varphi_{r}\right\}_{0}^{K}$ satisfying the conditions $\varphi_{0}=\varphi_{K}, \varphi_{1}-$ $\varphi_{0}=\varphi_{K}-\varphi_{K-1}$. With the help of $\mathbb{A}_{h}^{x}$, we arrive at the initial value problem

$$
\left\{\begin{array}{l}
\frac{d u^{h}(t, x)}{d t}+\mathbb{A}_{h}^{x} u^{h}(t, x)=b(t) \mathbb{A}_{h}^{x} u^{h}(t-\omega, x)+f^{h}(t, x), \quad t \geq 0, x \in[0, l]_{h}, \\
u^{h}(t, x)=g^{h}(t, x)=g(t, x) \quad(-\omega \leq t \leq 0), x \in[0, l]_{h}
\end{array}\right.
$$

for the system of ordinary differential equations. In the second step, problem (34) is replaced by the first-order accuracy of the difference scheme in $t$

$$
\left\{\begin{array}{l}
\frac{1}{\tau}\left(u_{k}^{h}(x)-u_{k-1}^{h}(x)\right)+\mathbb{A}_{h}^{x} u_{k}^{h}(x)=b\left(t_{k}\right) \mathbb{A}_{h}^{x} u_{k-N}^{h}(x)+f_{k}^{h}(x), \\
f_{k}^{h}(x)=f^{h}\left(t_{k}, x\right), \quad t_{k}=k \tau, 1 \leq k, N \tau=\omega, x \in[0, l]_{h}, \\
u_{k}^{h}(x)=g^{h}\left(t_{k}, x\right), \quad t_{k}=k \tau,-N \leq k \leq 0, x \in[0, l]_{h} .
\end{array}\right.
$$

Theorem 7 Assume that all the conditions of Theorem 5 are satisfied. Then for the solution of the difference scheme (35) the coercive stability estimate (31) holds.

The proof of Theorem 7 is based on the estimate

$$
\left\|e^{-t_{k} \mathbb{A}_{h}^{x}}\right\|_{C_{h} \rightarrow C_{h}} \leq M, \quad k \geq 0,
$$


and on the abstract Theorem 1 , the positivity of the operator $\mathbb{A}_{h}^{x}$ in $C_{h}^{\mu}$, and on the following theorem on the structure of the fractional space $E_{\alpha}\left(C_{h}, \mathbb{A}_{h}^{x}\right)$.

Theorem 8 For any $0<\alpha<\frac{1}{2}$ the norms in the spaces $E_{\alpha}\left(C_{h}, \mathbb{A}_{h}^{x}\right)$ and $C_{h}^{2 \alpha}$ are equivalent uniformly in $h$ [27].

Third, the initial value problem on the range

$$
\left\{0 \leq t \leq 1, x=\left(x_{1}, \ldots, x_{n}\right) \in \mathbb{R}^{n}, r=\left(r_{1}, \ldots, r_{n}\right)\right\}
$$

for $2 m$ th-order multidimensional delay differential equations of parabolic type is considered:

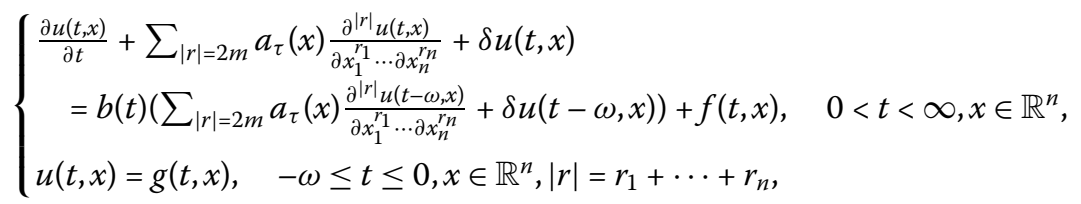

where $a_{r}(x), b(t), g(t, x), f(t, x)$ are given sufficiently smooth functions and $\delta>0$ is a sufficiently large number. We will assume that the symbol $\left[\xi=\left(\xi_{1}, \ldots, \xi_{n}\right) \in \mathbb{R}^{n}\right]$

$$
A_{1}^{x}(\xi)=\sum_{|r|=2 m} a_{r}(x)\left(i \xi_{1}\right)^{r_{1}} \cdots\left(i \xi_{n}\right)^{r_{n}}
$$

of the differential operator of the form

$$
A_{1}^{x}=\sum_{|r|=2 m} a_{r}(x) \frac{\partial^{|r|}}{\partial x_{1}^{r_{1}} \cdots \partial x_{n}^{r_{n}}},
$$

acting on the functions defined on the space $\mathbb{R}^{n}$, satisfies the inequalities

$$
0<M_{1}|\xi|^{2 m} \leq(-1)^{m} A_{1}^{x}(\xi) \leq M_{2}|\xi|^{2 m}<\infty
$$

for $\xi \neq 0$, where $|\xi|=\sqrt{\left|\xi_{1}\right|^{2}+\cdots+\left|\xi_{n}\right|^{2}}$. The discretization of problem (36) is carried out in two steps. In the first step the uniform grid space $\mathbb{R}_{h}^{n}\left(0<h \leq h_{0}\right)$ is defined as the set of all points of the Euclidean space $\mathbb{R}^{n}$ whose coordinates are given by

$$
x_{k}=s_{k} h, \quad s_{k}=0, \pm 1, \pm 2, \ldots, k=1, \ldots, n .
$$

The difference operator $A_{h}^{x}=B_{h}^{x}+\sigma I_{h}$ is assigned to the differential operator $A^{x}=B^{x}+\sigma I$, defined by equation (36). The operator

$$
B_{h}^{x}=h^{-2 m} \sum_{2 m \leq|s| \leq S} b_{s}^{x} \Delta_{1-}^{s_{1}} \Delta_{1+}^{s_{2}} \cdots \Delta_{n-}^{s_{2 n-1}} \Delta_{n+}^{s_{2 n}}
$$

acts on functions defined on the entire space $\mathbb{R}_{h}^{n}$. Here $s \in \mathbb{R}^{2 n}$ is a vector with nonnegative integer coordinates,

$$
\Delta_{k \pm} f^{h}(x)= \pm\left(f^{h}\left(x \pm e_{k} h\right)-f^{h}(x)\right)
$$

where $e_{k}$ is the unit vector of the axis $x_{k}$. 
An infinitely differentiable function $\varphi(x)$ of the continuous argument $x \in \mathbb{R}^{n}$ that is continuous and bounded together with all its derivatives is said to be smooth. We say that the difference operator $A_{h}^{x}$ is a $\lambda$ th-order $(\lambda>0)$ approximation of the differential operator $A^{x}$ if the inequality

$$
\sup _{x \in \mathbb{R}_{h}^{n}}\left|A_{h}^{x} \varphi(x)-A^{x} \varphi(x)\right| \leq M(\varphi) h^{\lambda}
$$

holds for any smooth function $\varphi(x)$. The coefficients $b_{s}^{x}$ are chosen in such a way that the operator $A_{h}^{x}$ approximates in a specified way the operator $A^{x}$. It will be assumed that the operator $A_{h}^{x}$ approximates the differential operator $A^{x}$ with any prescribed order [33, 34].

The function $A^{x}(\xi h, h)$ is obtained by replacing the operator $\Delta_{k \pm}$ in the right-hand side of the equality (38) with the expression $\pm\left(e^{ \pm i \xi_{k} h}-1\right)$, respectively, and it is called the symbol of the difference operator $B_{h}^{x}$.

It will be assumed that for $\left|\xi_{k} h\right| \leq \pi$ and fixed $x$ the symbol $A^{x}(\xi h, h)$ of the operator $B_{h}^{x}=A_{h}^{x}-\sigma I_{h}$ satisfies the inequalities

$$
(-1)^{m} A^{x}(\xi h, h) \geq M|\xi|^{2 m}, \quad\left|\arg A^{x}(\xi h, h)\right| \leq \phi<\phi_{0} \leq \frac{\pi}{2} .
$$

Suppose that the coefficient $b_{s}^{x}$ of the operator $B_{h}^{x}=A_{h}^{x}-\sigma I_{h}$ is bounded and satisfies the inequalities

$$
\left|b_{s}^{x+e_{k} h}-b_{s}^{x}\right| \leq M h^{\varepsilon}, \quad x \in \mathbb{R}_{h}^{n}, \varepsilon \in(0,1]
$$

With the help of $A_{h}^{x}$ we arrive at the initial value problem

$$
\left\{\begin{array}{l}
\left(u^{h}(t, x)\right)^{\prime}+A_{h}^{x} u^{h}(t, x)=b(t) A_{h}^{x} u^{h}(t-\omega, x)+f^{h}(t, x), \quad t \geq 0, x \in \mathbb{R}_{h}^{n}, \\
u^{h}(t, x)=g^{h}(t, x)=g(t, x) \quad(-\omega \leq t \leq 0), x \in \mathbb{R}_{h}^{n},
\end{array}\right.
$$

for an infinite system of ordinary differential equations. Now, problem (41) is replaced by the first-order accuracy of the difference scheme in $t$

$$
\left\{\begin{array}{l}
\frac{1}{\tau}\left(u_{k}^{h}(x)-u_{k-1}^{h}(x)\right)+A_{h}^{x} u_{k}^{h}(x)=b\left(t_{k}\right) A_{h}^{x} u_{k-N}^{h}(x)+f_{k}^{h}(x) \\
f_{k}^{h}(x)=f^{h}\left(t_{k}, x\right), \quad t_{k}=k \tau, 1 \leq k, N \tau=\omega, x \in \mathbb{R}_{h}^{n} \\
u_{k}^{h}(x)=g^{h}\left(t_{k}, x\right), \quad t_{k}=k \tau,-N \leq k \leq 0, x \in \mathbb{R}_{h}^{n} .
\end{array}\right.
$$

To formulate the result, one needs to introduce the spaces $C_{h}=C\left(\mathbb{R}_{h}^{n}\right)$ and $C_{h}^{\beta}=C^{\beta}\left(\mathbb{R}_{h}^{n}\right)$ of all bounded grid functions $u^{h}(x)$ defined on $\mathbb{R}_{h}^{n}$, equipped with the norms

$$
\begin{aligned}
& \left\|u^{h}\right\|_{C_{h}}=\sup _{x \in \mathbb{R}_{h}^{n}}\left|u^{h}(x)\right|, \\
& \left\|u^{h}\right\|_{C_{h}^{\beta}}=\sup _{x \in \mathbb{R}_{h}^{n}}\left|u^{h}(x)\right|+\sup _{x, y \in \mathbb{R}_{h}^{n}} \frac{\left|u^{h}(x)-u^{h}(x+y)\right|}{|y|^{\beta}} .
\end{aligned}
$$


Theorem 9 Assume that all the conditions of Theorem 7 are satisfied. Then for the solution of the difference scheme (42) the following coercive stability estimates hold:

$$
\begin{aligned}
& \left\|\tau^{-1}\left(u_{k}^{h}-u_{k-1}^{h}\right)\right\|_{C^{2 m \alpha}\left(\mathbb{R}_{h}^{n}\right)}+\sum_{2 m \leq|s| \leq S} h^{-2 m}\left\|\Delta_{1-}^{s_{1}} \Delta_{1+}^{s_{2}} \cdots \Delta_{n-}^{s_{2 n-1}} \Delta_{n+}^{s_{2 n} n} u_{k}^{h}\right\|_{C^{2 m \alpha}\left(\mathbb{R}_{h}^{n}\right)} \\
& \leq M_{2}(\alpha)\left[\max _{-N \leq i \leq 0} \sum_{2 m \leq|s| \leq S} h^{-2 m}\left\|\Delta_{1-}^{s_{1}} \Delta_{1+}^{s_{2}} \cdots \Delta_{n-}^{s_{2 n-1}} \Delta_{n+}^{s_{2 n}} g_{i}^{h}\right\|_{C^{2 m \alpha}\left(\mathbb{R}_{h}^{n}\right)}\right. \\
& \left.\quad+\sum_{n=1}^{\left[\frac{k}{N}\right]} \sup _{(n-1) N+1 \leq i \leq n N}\left\|f_{i}^{h}\right\|_{C^{2 m \alpha}\left(\mathbb{R}_{h}^{n}\right)}+\sup _{\left[\frac{k}{N}\right] N+1 \leq i \leq k}\left\|f_{i}^{h}\right\|_{C^{2 m \alpha}\left(\mathbb{R}_{h}^{n}\right)}\right], \quad 0<\alpha<\frac{1}{2 m}
\end{aligned}
$$

for all $k \geq 1$, where $M_{2}(\alpha)$ does not depend on $g_{k}^{h}$ and $f_{k}^{h}$.

The proof of Theorem 9 is based on the estimate

$$
\left\|e^{-t_{k} A_{h}^{x}}\right\|_{C\left(\mathbb{R}_{h}^{n}\right) \rightarrow C\left(\mathbb{R}_{h}^{n}\right)} \leq M, \quad k \geq 0,
$$

and on the abstract Theorem 1, the positivity of the operator $A_{h}^{x}$ in $C\left(\mathbb{R}_{h}^{n}\right)$, and on the fact that the $E_{\alpha}=E_{\alpha}\left(A_{h}^{x}, C\left(\mathbb{R}_{h}^{n}\right)\right)$ norms are equivalent to the norms $C^{2 m \alpha}\left(\mathbb{R}_{h}^{n}\right)$ uniformly in $h$ for $0<\alpha<\frac{1}{2 m}$ [23, Chapter 4, p.283].

\section{Conclusion}

In the present paper, the well-posedness of the difference schemes for the approximate solutions of the initial value problem for delay parabolic equations with unbounded operators acting on delay terms in an arbitrary Banach space is established. Theorems on the coercive stability of these difference schemes in fractional spaces are established. In practice, the coercive stability estimates in Hölder norms for the solutions of the difference schemes for the approximate solutions of the mixed problems for delay parabolic equations are obtained. Note that in the present paper $B(t)$ is a time variable unbounded space operator acting on the delay term. The delay $w$ is a positive constant. In general, it is interesting to consider the delay as a function $w(t)$, dependent on $t$. A well-known parabolic problem with delay used in population dynamics is the so-called Hutchinson equation where $B(t)$ is a time variable bounded nonlinear space operator acting on the delay term $[8,9]$. It would be interesting to consider the case when $B(t)$ is a nonlinear unbounded space operator acting on the delay term. Actually, it will be possible after establishing theorems on the existence, uniqueness, and stability of the solutions, and the smoothness property of the solutions, and obtaining a suitable contractivity condition of the numerical solutions.

Competing interests

The authors declare that they have no competing interests.

Authors' contributions

Each of the authors read and approved the final version of the manuscript.

Author details

${ }^{1}$ Department of Mathematics, Fatih University, Istanbul, 34500, Turkey. ${ }^{2}$ Department of Mathematics, ITTU, Gerogly

Street, Ashgabat, 74400, Turkmenistan. ${ }^{3}$ Department of Mathematics, Trakya University, Edirne, 22030, Turkey. 


\section{Acknowledgements}

This work is supported by Trakya University Scientific Research Projects Unit (Project No: 2010-91). We would like to thank to the reviewers, whose careful reading, helpful suggestions, and valuable comments helped us to improve the manuscript.

\section{Received: 8 November 2013 Accepted: 16 December 2013 Published: 16 January 2014}

\section{References}

1. Al-Mutib, AN: Stability properties of numerical methods for solving delay differential equations. J. Comput. Appl. Math. 10(1), 71-79 (1984)

2. Bellen, A: One-step collocation for delay differential equations. J. Comput. Appl. Math. 10(3), 275-283 (1984)

3. Cooke, KL, Györi, I: Numerical approximation of the solutions of delay differential equations on an infinite interval using piecewise constant arguments. Comput. Math. Appl. 28, 81-92 (1994)

4. Torelli, L: Stability of numerical methods for delay differential equations. J. Comput. Appl. Math. 25, 15-26 (1989)

5. Ashyralyev, A, Akca, H: On difference schemes for semilinear delay differential equations with constant delay. In: Proceeding of the Conference TSU: Actual Problems of Applied Mathematics, Physics and Engineering, Ashgabat, pp. 18-27 (1999)

6. Ashyralyev, A, Akca, H, Guray, U: Second order of accuracy difference scheme for approximate solutions of delay differential equations. Funct. Differ. Equ. 6(3-4), 223-231 (1999)

7. Wu, J: Theory and Applications of Partial Functional Differential Equations. Springer, New York (1996)

8. Higham, DJ, Sardar, TK: Existence and stability of fixed points for a discretised nonlinear reaction-diffusion equation with delay. Appl. Numer. Math. 18, 155-173 (1995)

9. Sardar, TK, Higham, DJ: Dynamics of constant and variable stepsize methods for a nonlinear population model with delay. Appl. Numer. Math. 24, 425-438 (1997)

10. Tanabe, H: Functional Analytic Methods for Partial Differential Equations. Dekker, New York (1997)

11. Akca, H, Shakhmurov, VB, Arslan, G: Differential-operator equations with bounded delay. Nonlinear Times Dig. 2 179-190 (1989)

12. Ashyralyev, A, Sobolevskii, PE: On the stability of the delay differential and difference equations. Abstr. Appl. Anal. 6(5), 267-297 (2001)

13. Ashyralyev, A, Sobolevskii, PE: New Difference Schemes for Partial Differential Equations. Birkhäuser, Basel (2004)

14. Di Blasio, G: Delay differential equations with unbounded operators acting on delay terms. Nonlinear Anal., Theory Methods Appl. 52(1), 1-18 (2003)

15. Ardito, A, Ricciardi, P: Existence and regularity for linear delay partial differential equations. Nonlinear Anal. 4, 411-414 (1980)

16. Sinestrari, E: On a class of retarded partial differential equations. Math. Z. 186, 223-224 (1984)

17. Ashyralyev, A, Agirseven, D: Finite difference method for delay parabolic equations. Numerical Analysis and Applied Mathematics ICNAAM 2011: International Conference on Numerical Analysis and Applied Mathematics. AIP Conf. Proc. 1389, 573-576 (2011)

18. Agirseven, D: Approximate solutions of delay parabolic equations with the Dirichlet condition. Abstr. Appl. Anal. 2012, Article ID 682752 (2012). doi:10.1155/2012/682752

19. Ashyralyev, A, Agirseven, D: On convergence of difference schemes for delay parabolic equations. Comput. Math. Appl. 66(7), 1232-1244 (2013)

20. Ashyralyev, A, Agirseven, D: Approximate solutions of delay parabolic equations with the Neumann condition. Numerical Analysis and Applied Mathematics ICNAAM 2012: International Conference on Numerical Analysis and Applied Mathematics. AIP Conf. Proc. 1479, 555-558 (2012). doi:10.1063/1.4756191

21. Sahmurova, A, Shakhmurov, VB: Parabolic problems with parameter occurring in environmental engineering. Book Editor(s): Ashyralyev, A; Lukashov, A. AIP Conf. Proc. 1470, 39-41 (2012)

22. Sahmurova, A, Shakhmurov, VB: Abstract parabolic problems with parameter and application. Appl. Math. Comput. 219(17), 9561-9571 (2013)

23. Ashyralyev, A, Sobolevskii, PE: Well-Posedness of Parabolic Difference Equations. Operator Theory Advances and Applications. Birkhäuser, Basel (1994)

24. Ashyralyev, A, Sobolevskii, PE: The theory of interpolation of linear operators and the stability of difference schemes. Dokl. Akad. Nauk SSSR 275(6), 1289-1291 (1984) (Russian)

25. Bazarov, MA: On the structure of fractional spaces. In: Proceedings of the XXVII All-Union Scientific Student Conference 'The Student and Scientific-Technological Progress', Novosibirsk. Gos. Univ., Novosibirsk, pp. 3-7 (1989) (Russian)

26. Triebel, H: Interpolation Theory, Function Spaces, Differential Operators. North-Holland, Amsterdam (1978)

27. Ashyralyev, A: Fractional spaces generated by the positive differential and difference operator in a Banach space. In: Tas, K, Tenreiro Machado, JA, Baleanu, D, (eds.) Proceedings of the Conference 'Mathematical Methods and Engineering', pp. 13-22. Springer, Netherlands (2007)

28. Ashyralyev, A, Akturk, S, Sozen, Y: Positivity of two-dimensional elliptic differential operators in Hölder spaces. AIP Conf. Proc. 1470, 77-79 (2012)

29. Ashyralyev, A, Yaz, N: On structure of fractional spaces generated by positive operators with the nonlocal boundary value conditions. In: Agarwal, RP, Perera, K (eds.) Proceedings of the Conference Differential and Difference Equations and Applications, pp. 91-101. Hindawi Publishing, New York (2006)

30. Ashyralyev, A, Tetikoğlu, FS: The structure of fractional spaces generated by the positive operator with periodic conditions. AIP Conf. Proc. 1470, 57-60 (2012)

31. Ashyralyev, A, Nalbant, N, Sozen, Y: Structure of fractional spaces generated by second order difference operators. J. Franklin Inst. (2013). doi:10.1016/j.jfranklin.2013.07.009

32. Ashyralyev, A, Akturk, S, Sozen, Y: The structure of fractional spaces generated by a two-dimensional elliptic differential operator and its applications. Bound. Value Probl. 2014, 3 (2014)

33. Smirnitskii, YA, Sobolevskii, PE: Positivity of multidimensional difference operators in the C-norm. Usp. Mat. Nauk 36(4), 202-203 (1981) (Russian) 
34. Smirnitskii, YA: Fractional powers of elliptic difference operators. PhD Thesis, Voronezh State University, Voronezh (1983) (Russian)

doi:10.1186/1687-1847-2014-18

Cite this article as: Ashyralyev and Agirseven: Well-posedness of delay parabolic difference equations. Advances in

Difference Equations 2014 2014:18.

Submit your manuscript to a SpringerOpen ${ }^{\circ}$ journal and benefit from:

- Convenient online submission

- Rigorous peer review

- Immediate publication on acceptance

- Open access: articles freely available online

- High visibility within the field

- Retaining the copyright to your article

Submit your next manuscript at $\boldsymbol{\wedge}$ springeropen.com 